\title{
Stability and Bifurcation for a Simply Supported Functionally Graded Material Plate with One-to-One Internal Resonance
}

\author{
Dongmei Zhang ${ }^{1,2}$ and Fangqi Chen ${ }^{3}$ \\ ${ }^{1}$ Department of Mechanics, Nanjing University of Aeronautics and Astronautics, Nanjing 210016, China \\ ${ }^{2}$ School of Science, Linyi University, Shandong 276005, China \\ ${ }^{3}$ Department of Mathematics, Nanjing University of Aeronautics and Astronautics, Nanjing 210016, China \\ Correspondence should be addressed to Dongmei Zhang; zhangdongmei_2008@163.com
}

Received 2 January 2014; Accepted 22 April 2014; Published 26 May 2014

Academic Editor: Xiao-Qiao He

Copyright (C) 2014 D. Zhang and F. Chen. This is an open access article distributed under the Creative Commons Attribution License, which permits unrestricted use, distribution, and reproduction in any medium, provided the original work is properly cited.

\begin{abstract}
Stability and bifurcation behaviors for a model of simply supported functionally graded materials rectangular plate subjected to the transversal and in-plane excitations are studied by means of combination of analytical and numerical methods. The resonant case considered here is 1:1 internal resonances and primary parametric resonance. Two types of degenerated equilibrium points are studied in detail, which are characterized by a double zero and two negative eigenvalues, and a double zero and a pair of pure imaginary eigenvalues. For each case, the stability regions of the initial equilibrium solution and the critical bifurcation curves are obtained in terms of the system parameters which may lead to Hopf bifurcation and 2D torus. With both analytical and numerical methods, bifurcation behaviors on damping parameters and detuning parameters are studied, respectively. A time integration scheme is used to find the numerical solutions for these bifurcation cases, and numerical results agree with the analytic predictions.
\end{abstract}

\section{Introduction}

Functionally graded materials (FGMs) are extremely excellent engineering composite materials. Because of their specially tailored thermomechanical properties, FGMs are widely applied to large space station, shuttle, aircraft, automotive, and many others [1-3]. With the increasing use of FGM plates in engineering fields, research on the nonlinear dynamics of the FGM plates plays a significant role in applications. Holmes [4] studied flow-induced vibrations, bifurcations, and the chaos of thin plates. Yang and Sethna [5] used the averaging method to study the local and global bifurcations for parametrically excited vibrations of nearly square plates. Yu et al. [6] investigated the nonlinear vibrations and bifurcations of a thin plate with the aid of computation of normal form. Ye et al. [7] investigated the nonlinear vibrations and chaotic dynamics of an ant symmetric crossply laminated composite rectangular thin plate under parametric excitation. Zhang et al. [8] studied the bifurcation and chaotic motions of a composite laminated piezoelecttric rectangular plate with 1:2 internal resonance. Chang et al.
[9] investigated the bifurcations and chaos of a rectangular thin plate with 1:1 internal resonance. Zhang et al. [10] studied the nonlinear dynamics of a parametrically excited simply supported laminated composite plate and found that there exist the multiple steady bifurcation solutions under the certain conditions.

Using asymptotic perturbation method based on the Fourier expansion and the temporal rescaling [11, 12], Zhang et al. [13] investigated the nonlinear oscillations and chaotic dynamics of a simply supported FGM plate. Yu and Huseyin $[14,15]$ studied the stability and bifurcation behaviors of the systems which were characterized by double zero eigenvalues, a simple pair of pure imaginary eigenvalues, and two pairs of pure imaginary eigenvalues. Yu [16] used a perturbation technique and computer algebra to compute the normal form of the nonlinear dynamical systems with two pairs of pure imaginary eigenvalues and analyzed double Hopf bifurcation. Hao et al. [17] analyzed the nonlinear dynamics of a simply supported FGM plate subjected to the transverse and in-plane excitations in a thermal environment. Zhang et al. [18] studied the stability and bifurcation 


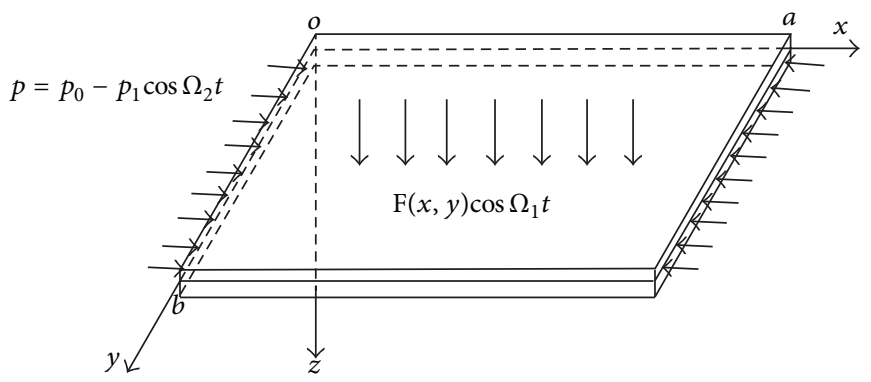

FIGURE 1: The model of a rectangular FGM plate and the coordinate system.

behaviors of FGM which were characterized by a simple zero and a pair of purely imaginary eigenvalues and two pairs of pure imaginary eigenvalues. By the analytical approach, the present work investigates theoretically the stability conditions of the equilibrium solutions and their stability boundaries.

The objective of this paper is to investigate the local dynamic behaviors of a simply supported rectangular plate subjected to the transversal and in-plane excitations in a thermal environment. The resonant case considered here is 1:1 internal resonance and primary parametric resonance. Both analytical and numerical approaches are employed to consider the bifurcation and stability of this system. Two types of degenerated equilibrium points are studied in detail, which are characterized by a double zero and two negative eigenvalues and a double zero and a pair of pure imaginary eigenvalues, respectively. The stability regions of the initial equilibrium solution and the critical bifurcation curves are obtained in terms of the system parameters. Bifurcations leading to Hopf bifurcation and 2D torus and their stability conditions are also investigated. All numerical results agree with the analytic predictions.

This paper is organized as follows. In Section 2, the averaged equations of transverse motion of the FGM plate are given and the stability conditions of initial equilibrium solution are obtained explicitly. Section 3 is devoted to the studies on the dynamical behaviors of the system in the vicinity of the critical points: a double zero and two negative eigenvalues and a double zero and a pair of pure eigenvalues. Finally some conclusions are drawn in Section 4.

\section{Formulation of the Problem}

The paper focuses on the stability and bifurcation behaviors of a functionally graded material rectangular plate subjected to the in-plane and transversal excitations. The model is shown in Figure 1.

The paper focuses on the stability and bifurcations behaviors of a simply supported FGM rectangular under combined parametric and external excitations. The model is shown in Figure 1. The plate is of length $a$, width $b$, and thickness $h$. Let $(u, v, w)$ and $\left(u_{0}, v_{0}, w_{0}\right)$ be the displacements of an arbitrary point and a point in the middle plane of the plate in the $x, y$, and $z$ directions; $\varphi_{x}$ and $\varphi_{y}$ represent the mid-plane rotations of a transverse normal about the $y$ and $x$ axes. The plate is subjected to an in-plane excitation $p=p_{0}-p_{1} \cos \Omega_{2} t$ uniformly distributed along the plate edges $x=0, x=a$, and a transverse excitation $F(x, y) \cos \Omega_{1} t$. Here $\Omega_{1}$ and $\Omega_{2}$ are the excitation frequencies of the transverse and in-plane excitations, respectively.

Applying the Galerkin procedure, the nonlinear dimensionless governing differential equations of transverse motion of the FGM rectangular plate are obtained as follows [17]:

$$
\begin{aligned}
\ddot{w}_{1}+ & \omega_{1}^{2} w_{1}+a_{1} \dot{w}_{1}+a_{2} w_{1} \cos \Omega_{2} t+a_{3} w_{1}^{2} \\
& +a_{4} w_{2}^{2}+a_{5} w_{1} w_{2}^{2}+a_{6} w_{1}^{3}+a_{7} w_{1} w_{2} \\
= & f_{1} \cos \Omega_{1} t \\
\ddot{w}_{2}+ & \omega_{2}^{2} w_{2}+b_{1} \dot{w}_{2}+b_{2} w_{2} \cos \Omega_{2} t+b_{4} w_{1}^{2} \\
& +b_{5} w_{2}^{2}+b_{6} w_{2} w_{1}^{2}+b_{7} w_{2}^{3}+b_{3} w_{1} w_{2} \\
= & f_{2} \cos \Omega_{1} t .
\end{aligned}
$$

It is assumed that the width-to-length ratio of the FGM rectangular plate is $a / b=1$. Therefore only the primary parametric resonance and 1:1 internal resonance are considered in this paper. In this resonant case, there are the following relations:

$$
\omega_{1}=\frac{\Omega}{2}+\epsilon^{2} \sigma_{1}, \quad \omega_{2}=\frac{\Omega}{2}+\epsilon^{2} \sigma_{2}, \quad \Omega_{1}=\Omega_{2}=\Omega,
$$

where $\epsilon$ is a small perturbation parameter, $\omega_{1}$ and $\omega_{2}$ are the first-order and second-order linear frequencies, and $\sigma_{1}$ and $\sigma_{2}$ are two detuning parameters. Using the asymptotic perturbation method, the averaged equation in the Cartesian form is obtained as follows [17]:

$$
\begin{aligned}
& \dot{x}_{1}=\mu_{1} x_{1}+\left(\sigma_{1}+\alpha\right) x_{2}+k_{1} x_{4}+N f_{1}, \\
& \dot{x}_{2}=\left(-\sigma_{1}+\alpha\right) x_{1}+\mu_{1} x_{2}+k_{1} x_{3}+N f_{2}, \\
& \dot{x}_{3}=k_{2} x_{2}+\mu_{2} x_{3}+\left(\sigma_{2}+\beta\right) x_{4}+N f_{3}, \\
& \dot{x}_{4}=k_{2} x_{1}+\left(-\sigma_{2}+\beta\right) x_{3}+\mu_{2} x_{4}+N f_{4},
\end{aligned}
$$

where $\alpha=a_{1}+a_{2} f_{1}+a_{11} f_{2}, \beta=b_{1}+b_{2} f_{1}+b_{3} f_{2}, k_{1}=$ $a_{3} f_{2}+a_{11} f_{1}$, and $k_{2}=b_{4} f_{2}+b_{5} f_{1}$, where all coefficients can be found in [17]. The nonlinear functions $N f_{i}(i=1,2,3,4)$ are presented in the appendix. 
The Jacobian matrix of (3a)-(3d) evaluated at the initial equilibrium solution $\left(x_{1}, x_{2}, x_{3}, x_{4}\right)=(0,0,0,0)$ is as follows:

$$
\mathbf{J}=\left(\begin{array}{cccc}
\mu_{1} & \sigma_{1}+\alpha & 0 & k_{1} \\
-\sigma_{1}+\alpha & \mu_{1} & k_{1} & 0 \\
0 & k_{2} & \mu_{2} & \sigma_{2}+\beta \\
k_{2} & 0 & -\sigma_{2}+\beta & \mu_{2}
\end{array}\right)
$$

From which one may obtain the characteristic polynomial

$$
f(\lambda)=\lambda^{4}+b_{1} \lambda^{3}+b_{2} \lambda^{2}+b_{3} \lambda+b_{4},
$$

where

$$
\begin{aligned}
b_{1}= & -2 \mu_{1}-2 \mu_{2}, \\
b_{2}= & \mu_{2}^{2}+4 \mu_{1} \mu_{2}+\sigma_{1}^{2}-\alpha^{2}+\mu_{1}^{2}+\sigma_{2}^{2}-\beta^{2}-2 k_{1} k_{2}, \\
b_{3}= & -2 \sigma_{2}^{2} \mu_{1}-2 \mu_{2}^{2} \mu_{1}-2 \sigma_{1}^{2} \mu_{2}-2 \alpha^{2} \mu_{2} \\
& -2 \mu_{1}^{2} \mu_{2}+2 k_{1} k_{2} \mu_{1}+2 k_{1} k_{2} \mu_{2} \\
& +2 \mu_{1} \beta^{2}+2 \mu_{2} \alpha^{2} \\
b_{4}= & \sigma_{1}^{2} \sigma_{2}^{2}-\sigma_{2}^{2} \alpha^{2}+\sigma_{2}^{2} \mu_{1}^{2}+\mu_{2}^{2} \sigma_{1}^{2} \\
& -2 k_{1} k_{2} \sigma_{1} \sigma_{2}-2 k_{1} k_{2} \alpha \beta \\
& -2 k_{1} k_{2} \mu_{1} \mu_{2}+\alpha^{2} \beta^{2}-\mu_{2}^{2} \alpha^{2}+\mu_{1}^{2} \mu_{2}^{2} \\
& -\sigma_{1}^{2} \beta^{2}+k_{1}^{2} k_{2}^{2}-\mu_{1}^{2} \beta^{2} .
\end{aligned}
$$

By the Routh-Hurwitz criterion, the equilibrium solution $\left(x_{1}, x_{2}, x_{3}, x_{4}\right)=(0,0,0,0)$ is stable, if the following conditions are satisfied:

$$
\begin{aligned}
& b_{1}>0, \quad b_{1} b_{2}-b_{3}>0, \quad b_{4}>0, \\
& b_{3}\left(b_{1} b_{2}-b_{3}\right)-b_{1}^{2} b_{4}>0
\end{aligned}
$$

Conditions in (7) imply that all the eigenvalues of the Jacobian matrix (4) have negative real parts. When conditions in (7) are not satisfied, the initial equilibrium solution is unstable, and bifurcation may occur. In the next section, the detailed analysis will be given when conditions in (7) are violated.

\section{Stability and Bifurcation Behaviors}

3.1. Stability and Bifurcation Behaviors on the Damping Parameters. In this Section, the stability and bifurcation analysis on the parameters $\mu_{1}$ and $\mu_{2}$ are investigated, which can be divided into two cases due to degenerated equilibrium points.

3.1.1. The Case of Double Zero and Two Negative Eigenvalues. In this part, the characteristic polynomial (5) is supposed to have a double zero and two negative eigenvalues, $\lambda_{1,2}=$ $0, \lambda_{3,4}=-2$, which implies that $b_{1}=b_{2}=4, b_{3}=b_{4}=0$. One choice of the parameters that satisfy these conditions is $\mu_{1}=-1, \mu_{2}=-1, \sigma_{1}=1, \sigma_{2}=1, \alpha=0, \beta=0, k_{1}=1$, $k_{2}=2, a_{4}=a_{5}=a_{6}=a_{7}=a_{8}=a_{9}=a_{10}=1$, $b_{6}=b_{7}=b_{8}=b_{9}=b_{10}=b_{11}=1$. Choosing $\mu_{1}$ and $\mu_{2}$ as perturbation parameters and using the parameter transformation $\mu_{1}=-1+\delta_{1}, \mu_{2}=-1+\delta_{2}$ and the state variable transformation

$$
\left[\begin{array}{l}
x_{1} \\
x_{2} \\
x_{3} \\
x_{4}
\end{array}\right]=\left[\begin{array}{cccc}
\frac{1}{2} & \frac{1}{2} & -\frac{1}{2} & \frac{1}{2} \\
-\frac{1}{2} & \frac{1}{2} & -\frac{1}{2} & -\frac{1}{2} \\
0 & 1 & 0 & 1 \\
1 & 0 & 1 & 0
\end{array}\right]\left[\begin{array}{l}
z_{1} \\
z_{2} \\
z_{3} \\
z_{4}
\end{array}\right]
$$

one may transform (3a)-(3d) into a new system as follows:

$$
\begin{aligned}
\dot{z}_{1}= & \frac{1}{2}\left(\delta_{1}+\delta_{2}\right) z_{1}+\frac{1}{2}\left(\delta_{1}-\delta_{2}\right) z_{2}-\frac{1}{2}\left(\delta_{1}-\delta_{2}\right) z_{3} \\
& +\frac{1}{2}\left(\delta_{1}-\delta_{2}\right) z_{4}+N g_{1}, \\
\dot{z}_{2}= & -\frac{1}{2}\left(\delta_{1}-\delta_{2}\right) z_{1}+\frac{1}{2}\left(\delta_{1}+\delta_{2}\right) z_{2}-\frac{1}{2}\left(\delta_{1}-\delta_{2}\right) z_{3} \\
& -\frac{1}{2}\left(\delta_{1}-\delta_{2}\right) z_{4}+N g_{2}, \\
\dot{z}_{3}= & -2 z_{3}-\frac{1}{2}\left(\delta_{1}-\delta_{2}\right) z_{1}-\frac{1}{2}\left(\delta_{1}-\delta_{2}\right) z_{2} \\
& +\frac{1}{2}\left(\delta_{1}+\delta_{2}\right) z_{3}-\frac{1}{2}\left(\delta_{1}-\delta_{2}\right) z_{4}+N g_{3}, \\
\dot{z}_{4}= & -2 z_{4}+\frac{1}{2}\left(\delta_{1}-\delta_{2}\right) z_{1}-\frac{1}{2}\left(\delta_{1}-\delta_{2}\right) z_{2} \\
& +\frac{1}{2}\left(\delta_{1}-\delta_{2}\right) z_{3}+\frac{1}{2}\left(\delta_{1}+\delta_{2}\right) z_{4}+N g_{4},
\end{aligned}
$$

where the nonlinear functions $N g_{i}(i=1, \ldots, 4)$ are exhibited in the appendix.

The Jacobian matrix of (9a)-(9d) evaluated at the initial equilibrium solution $\left(z_{1}, z_{2}, z_{3}, z_{4}\right)=(0,0,0,0)$ at critical point $\delta_{1 c}=\delta_{2 c}=0$ is the following canonical form:

$$
\mathbf{J}_{\left(z_{i}=0\right)}=\left[\begin{array}{cccc}
0 & 0 & 0 & 0 \\
0 & 0 & 0 & 0 \\
0 & 0 & -2 & 0 \\
0 & 0 & 0 & -2
\end{array}\right] .
$$

The local dynamic behaviors of system (9a)-(9d) are characterized by the critical variables $z_{1}$ and $z_{2}$. Furthermore, the bifurcation solutions for the noncritical variables $z_{3}$ and $z_{4}$ may be determined from (9a)-(9d) up to leading orders terms as

$$
\begin{aligned}
z_{3}= & -\frac{1}{4}\left(\delta_{1}-\delta_{2}\right) z_{1}-\frac{1}{4}\left(\delta_{1}-\delta_{2}\right) z_{2} \\
& -\frac{1}{4} z_{1}^{3}-\frac{9}{8} z_{2}^{3}-\frac{7}{4} z_{2}^{2} z_{1}-\frac{5}{8} z_{1}^{2} z_{2} \\
z_{4}= & \frac{1}{4}\left(\delta_{1}-\delta_{2}\right) z_{1}-\frac{1}{4}\left(\delta_{1}-\delta_{2}\right) z_{2}-\frac{3}{8} z_{1}^{3} \\
& +\frac{5}{2} z_{2}^{3}+z_{2}^{2} z_{1}+\frac{25}{8} z_{1}^{2} z_{2} .
\end{aligned}
$$




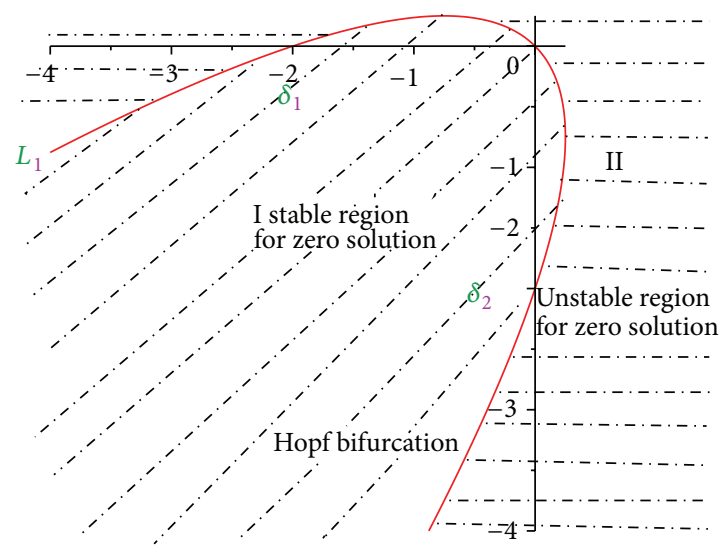

Figure 2: Bifurcation diagram on parameters $\mu_{1}$ and $\mu_{2}$ for the case of double zero and two negative eigenvalues.

In order to study the bifurcation and stable properties of system (9a)-(9d) in the vicinity of the critical point, one only needs to analyze the following two-dimensional system:

$$
\begin{aligned}
\dot{z}_{1}= & {\left[\frac{1}{2}\left(\delta_{1}+\delta_{2}\right)+\frac{1}{4}\left(\delta_{1}-\delta_{2}\right)^{2}\right] z_{1}+\frac{1}{2}\left(\delta_{1}-\delta_{2}\right) z_{2} } \\
& +\frac{3}{4} z_{1}^{3}-\frac{1}{2} z_{2}^{3}+\frac{1}{2} z_{1}^{2} z_{2}-\frac{1}{4} z_{2}^{2} z_{1}, \\
\dot{z}_{2}= & -\frac{1}{2}\left(\delta_{1}-\delta_{2}\right) z_{1}+\left[\frac{1}{2}\left(\delta_{1}+\delta_{2}\right)+\frac{1}{4}\left(\delta_{1}-\delta_{2}\right)^{2}\right] z_{2} \\
& +z_{1}^{3}-\frac{17}{4} z_{2}^{3}-\frac{5}{4} z_{1}^{2} z_{2}-4 z_{2}^{2} z_{1} .
\end{aligned}
$$

To find the stability conditions of the initial equilibrium solution $\left(z_{1}, z_{2}\right)=(0,0)$, one may evaluate the Jacobian matrix of (1la) and (11b) at $\left(z_{1}, z_{2}\right)=(0,0)$ and obtain

$$
\mathbf{J}_{\left(z_{i}=0\right)}=\left[\begin{array}{ll}
a_{11} & a_{12} \\
a_{21} & a_{21}
\end{array}\right]
$$

where

$$
\begin{aligned}
& a_{11}=\frac{1}{2}\left(\delta_{1}+\delta_{2}\right)+\frac{1}{4}\left(\delta_{1}-\delta_{2}\right)^{2}, \\
& a_{12}=\frac{1}{2}\left(\delta_{1}-\delta_{2}\right), \\
& a_{21}=-\frac{1}{2}\left(\delta_{1}-\delta_{2}\right), \\
& a_{22}=\frac{1}{2}\left(\delta_{1}+\delta_{2}\right)+\frac{1}{4}\left(\delta_{1}-\delta_{2}\right)^{2} .
\end{aligned}
$$

The characteristic polynomial is

$$
\begin{aligned}
f(\lambda)= & \lambda^{2}+\left[-\left(\delta_{1}+\delta_{2}\right)-\frac{1}{2}\left(\delta_{1}-\delta_{2}\right)^{2}\right] \lambda \\
& +\left[\left[\frac{1}{2}\left(\delta_{1}+\delta_{2}\right)+\frac{1}{4}\left(\delta_{1}-\delta_{2}\right)^{2}\right]^{2}+\frac{1}{4}\left(\delta_{1}-\delta_{2}\right)^{2}\right] .
\end{aligned}
$$

The stability conditions for the initial equilibrium solution $\left(z_{1}, z_{2}\right)=(0,0)$ are

$$
\begin{gathered}
-\left(\delta_{1}+\delta_{2}\right)-\frac{1}{2}\left(\delta_{1}-\delta_{2}\right)^{2}>0 \\
{\left[\frac{1}{2}\left(\delta_{1}+\delta_{2}\right)+\frac{1}{4}\left(\delta_{1}-\delta_{2}\right)^{2}\right]^{2}+\frac{1}{4}\left(\delta_{1}-\delta_{2}\right)^{2}>0 .}
\end{gathered}
$$

It is easy to see that $\left[(1 / 2)\left(\delta_{1}+\delta_{2}\right)+(1 / 4)\left(\delta_{1}-\delta_{2}\right)^{2}\right]^{2}+$ $(1 / 4)\left(\delta_{1}-\delta_{2}\right)^{2}>0$ unless $\left(\delta_{1}, \delta_{2}\right)=(0,0)$, so if $-\left(\delta_{1}+\delta_{2}\right)-$ $(1 / 2)\left(\delta_{1}-\delta_{2}\right)^{2}>0$, the initial equilibrium solution is stable. Then a critical bifurcation curve is obtained:

$$
L_{1}:\left(\delta_{1}+\delta_{2}\right)+\frac{1}{2}\left(\delta_{1}-\delta_{2}\right)^{2}=0 .
$$

When $\left(\delta_{1}, \delta_{2}\right) \in L_{1}$, Hopf bifurcation may occur. In fact, the eigenvalues of the Jacobian matrix at the initial equilibrium solution $\left(z_{1}, z_{2}, z_{3}, z_{4}\right)=(0,0,0,0)$ are

$$
\lambda_{1,2}=\left(\delta_{1}+\delta_{2}\right)+\frac{1}{2}\left(\delta_{1}-\delta_{2}\right)^{2} \pm \frac{\sqrt{2}}{4}\left(\delta_{1}-\delta_{2}\right) i .
$$

Let

$$
\begin{aligned}
& a=\operatorname{Re}\left(\lambda_{1,2}\right)=\left(\delta_{1}+\delta_{2}\right)+\frac{1}{2}\left(\delta_{1}-\delta_{2}\right)^{2}, \\
& b=\operatorname{Im}\left(\lambda_{1,2}\right)=\frac{\sqrt{2}}{4}\left(\delta_{1}-\delta_{2}\right) i .
\end{aligned}
$$

It is easy to see that $a=0, b>0$, and $d a / d \delta_{1} \neq 0$, when $\left(\delta_{1}, \delta_{2}\right) \in L_{1}$ and $\left(\delta_{1}, \delta_{2}\right) \neq 0$ or $(-3 / 4,1 / 4)$. By Hopf bifurcation theorem, along $L_{1}$, Hopf bifurcation may occur. The bifurcation diagram is shown in Figure 2. The initial equilibrium solution $\left(z_{1}, z_{2}, z_{3}, z_{4}\right)=(0,0,0,0)$ is stable while $\left(\delta_{1}, \delta_{2}\right)$ belongs to area I. Along $L_{1}$, Hopf bifurcation may occur. When $\left(\delta_{1}, \delta_{2}\right)$ crosses $L_{1}$ and goes into area II, the initial equilibrium solution becomes unstable.

Numercial results have been obtained by using a time integration scheme-fourth-order Runge-Kutta method. The numerical computation is performed on the base of the original differential equations (3a)-(3d). For the parameter 


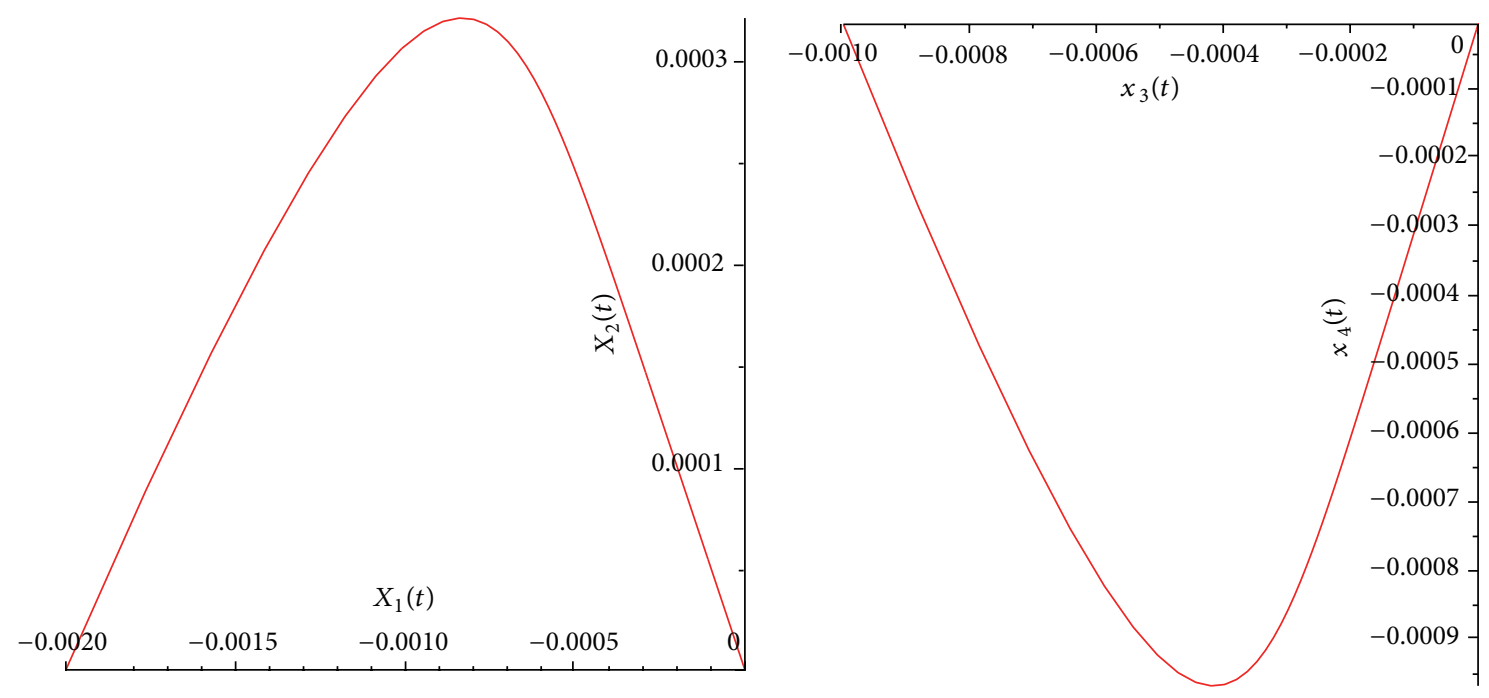

FIgURE 3: Trajectory projection starting from $\left(x_{1}, x_{2}, x_{3}, x_{4}\right)=(-0.002,0,-0.001,0)$ converges to the E.S. when $\left(\delta_{1}, \delta_{2}\right)=(-0.3,-0.3)$.
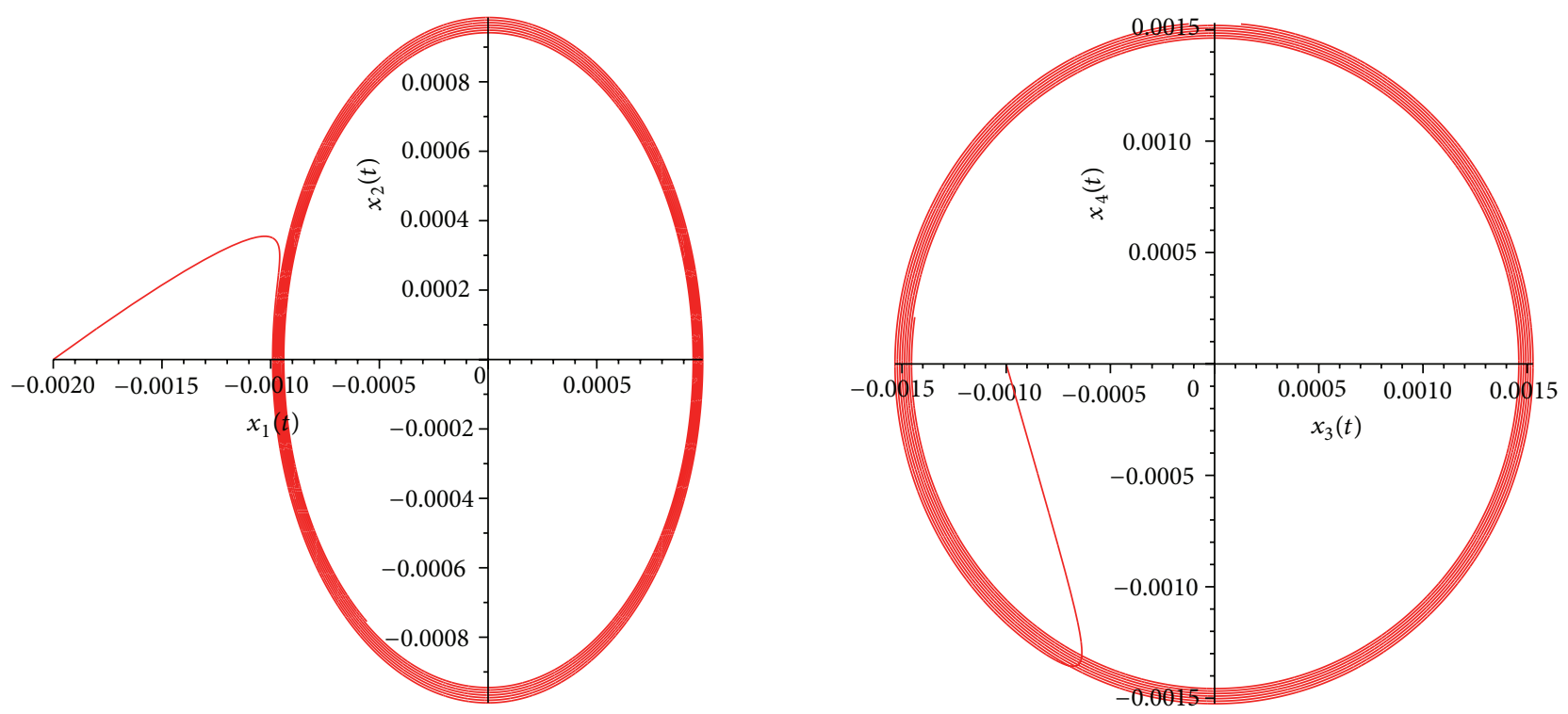

Figure 4: Trajectory projection starting from $\left(x_{1}, x_{2}, x_{3}, x_{4}\right)=(-0.002,0,-0.001,0)$ converges to the E.S. when $\left(\xi_{1}, \xi_{2}\right)=(-0.1,0.08321)$.

$\left(\delta_{1}, \delta_{2}\right)=(0.2,-0.3)$, which is located in the area II (the unstable region of the initial equilibrium solution), numerical results show that any solution starting from an arbitrary initial point $\left(x_{1}, x_{2}, x_{3}, x_{4}\right) \neq(0,0,0,0)$ diverges to infinity, initiating that the initial equilibrium solution is unstable, as predicted by the analytic study. When the parameter value is chosen from the stable region of the E.S., say, $\left(\delta_{1}, \delta_{2}\right)=$ $(-0.3,-0.3)$, a numerical solution starting from an initial point $\left(x_{1}, x_{2}, x_{3}, x_{4}\right)=(-0.002,0,-0.001,0)$ is obtained, which converges to the origin, implying that the E.S. is stable. This is shown in Figure 3, where the phase trajectories are projected onto the $x_{1}-x_{2}$ and $x_{3}-x_{4}$ plane. It should be noted that since the study is focused on the local dynamic behaviors of the FGM in the vicinity of a critical point, so the parameter $\left(\delta_{1}, \delta_{2}\right)$ should be chosen near the critical point $\left(\delta_{1}, \delta_{2}\right)=(0,0)$.
If one chooses $\left(\delta_{1}, \delta_{2}\right)=(-0.1,0.08321)$, which is located in the curve $L_{1}$, Hopf bifurcation may occur. The phase trajectories starting from $\left(x_{1}, x_{2}, x_{3}, x_{4}\right)=(-0.002,0,-0.001,0)$ are shown in Figure 4.

3.1.2. Double Zero and a Pair of Pure Imaginary Eigenvalues. Choosing the following parameter values: $\mu_{1}=1, \mu_{2}=-1$, $\sigma_{1}=1, \sigma_{2}=-1, \alpha=0, \beta=0, k_{1}=1, k_{2}=-2, a_{4}=a_{5}=a_{6}=$ $a_{7}=a_{8}=a_{9}=a_{10}=1, b_{6}=b_{7}=b_{8}=b_{9}=b_{10}=b_{11}=1$, which imply that $b_{2}=4, b_{1}=b_{3}=b_{4}=0$, then the Jacobian matrix (4) has the eigenvalues $\lambda_{1,2}=0, \lambda_{3,4}= \pm 2 i$.

Choosing $\mu_{1}$ and $\mu_{2}$ as perturbation parameters, and using the parameter transformation $\mu_{1}=1+\delta_{1}, \mu_{2}=-1+\delta_{2}$, the characteristic polynomial (5) of the Jacobian matrix (4) becomes

$$
\tilde{f}(\lambda)=\lambda^{4}+\widetilde{b}_{1} \lambda^{3}+\widetilde{b}_{2} \lambda^{2}+\widetilde{b}_{3} \lambda+\widetilde{b}_{4}
$$


where,

$$
\begin{aligned}
& \widetilde{b}_{1}=-2 \delta_{1}-2 \delta_{2}, \\
& \widetilde{b}_{2}=\delta_{1}^{2}+\delta_{2}^{2}+4 \delta_{1} \delta_{2}-2 \delta_{1}+2 \delta_{2}+1, \\
& \widetilde{b}_{3}=-2 \delta_{1} \delta_{2}^{2}-2 \delta_{2} \delta_{1}^{2}+2 \delta_{1}^{2}-2 \delta_{2}^{2}-4 \delta_{1}-4 \delta_{2}, \\
& \widetilde{b}_{4}=2 \delta_{1}^{2}+2 \delta_{2}^{2}+\delta_{2}^{2} \delta_{1}^{2}-2 \delta_{2} \delta_{1}^{2}+2 \delta_{1} \delta_{2}^{2} .
\end{aligned}
$$

The stability conditions for the initial equilibrium solution $\left(x_{1}, x_{2}, x_{3}, x_{4}\right)=(0,0,0,0)$ are

$$
\begin{aligned}
& \Delta_{1}=\widetilde{b}_{1}>0 \text {, that is }-2 \delta_{1}-2 \delta_{2}>0, \\
& \Delta_{2}=\widetilde{b}_{1} \widetilde{b}_{2}-\widetilde{b}_{3}>0, \\
& \text { that is }-2\left(\delta_{1}+\delta_{2}\right)\left(\delta_{1}^{2}+\delta_{2}^{2}+3 \delta_{1} \delta_{2}\right. \\
& \left.\qquad-\delta_{1}+\delta_{2}+2\right)>0, \\
& \Delta_{3}=\widetilde{b}_{3}\left(\widetilde{b}_{1} \widetilde{b}_{2}-\widetilde{b}_{3}\right)-\widetilde{b}_{1}^{2} \widetilde{b}_{4}, \\
& \text { that is } 4\left(\delta_{1}+\delta_{2}\right)^{2}\left(\delta_{2}^{2}+\delta_{1}^{2}+2 \delta_{1} \delta_{2}+4\right) \\
& \qquad \times\left(\delta_{2}-\delta_{1}+\delta_{1} \delta_{2}+1\right)>0 .
\end{aligned}
$$

From the four inequalities above, one may get the following three transition curves:

$$
\begin{aligned}
& L_{2}: \delta_{1}+\delta_{2}=0, \\
& L_{3}: \delta_{1}^{2}+\delta_{2}^{2}+3 \delta_{1} \delta_{2}-\delta_{1}+\delta_{2}+2=0, \\
& L_{4}:\left(\delta_{2}^{2}+\delta_{1}^{2}+2 \delta_{1} \delta_{2}+4\right)\left(\delta_{2}-\delta_{1}+\delta_{1} \delta_{2}+1\right)=0 .
\end{aligned}
$$

Then, the transition curves are shown in Figure 5.

When $\delta_{1}+\delta_{2}<0, \delta_{1}^{2}+\delta_{2}^{2}+3 \delta_{1} \delta_{2}-\delta_{1}+\delta_{2}+2>0$ and $\left(\delta_{2}^{2}+\delta_{1}^{2}+2 \delta_{1} \delta_{2}+4\right)\left(\delta_{2}-\delta_{1}+\delta_{1} \delta_{2}+1\right)>0$ are all satisfied, the initial equilibrium solution is stable. When $\left(\delta_{1}, \delta_{2}\right)$ belongs to region I, the initial equilibrium solution is stable, and when $\left(\delta_{1}, \delta_{2}\right)$ belongs to region II, the initial equilibrium solution of the system is unstable.

Similar to the case in Section 3.1, different parameters are chosen to conform the analytical results obtained in this Section. When the parameter is chosen as $\left(\delta_{1}, \delta_{2}\right)=$ $(-0.2,-0.2)$, numerical results show that a trajectory starting from $\left(x_{1}, x_{2}, x_{3}, x_{4}\right)=(-0.02,0.01,0.01,0.01)$ converges to the origin, implying that the E.S. is stable. The phase trajectories are projected onto the $x_{1}-x_{2}$ and $x_{3}-x_{4}$ subspaces as shown in Figure 6.

3.2. Stability and Bifurcation Behaviors on the Detuning Parameters. In this section, the stability and bifurcation analysis on the parameters $\sigma_{1}$ and $\sigma_{2}$ are investigated, which can be divided into two cases due to degenerated equilibrium points.

3.2.1. The Case of Double Zero and Two Negative Eigenvalues. Corresponding to Section 3.1.1, take parameter values $\mu_{1}=$

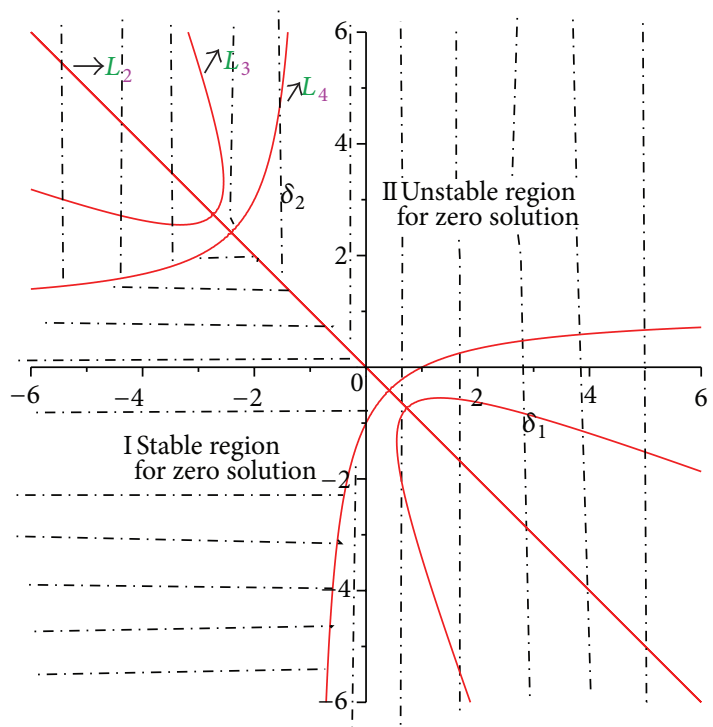

Figure 5: Bifurcation diagram on parameters $\mu_{1}$ and $\mu_{2}$ for the case of double zero and a pair of purely imaginary eigenvalues.

$-1, \mu_{2}=-1, \sigma_{1}=1, \sigma_{2}=1, \alpha=0, \beta=0, k_{1}=1, k_{2}=2$, $a_{4}=a_{5}=a_{6}=a_{7}=a_{8}=a_{9}=a_{10}=1, b_{6}=b_{7}=b_{8}=b_{9}=$ $b_{10}=b_{11}=1$.

Choosing $\sigma_{1}$ and $\sigma_{2}$ as perturbation parameters, and then using the parameter transformation $\sigma_{1}=1+\xi_{1}, \sigma_{2}=1+\xi_{2}$ and the state variable transformation (7), one can get

$$
\begin{aligned}
\dot{z}_{1}= & -\frac{1}{2}\left(\xi_{1}+\xi_{2}\right) z_{1}+\frac{1}{2}\left(\xi_{1}-\xi_{2}\right) z_{2} \\
& -\frac{1}{2}\left(\xi_{1}+\xi_{2}\right) z_{3}-\frac{1}{2}\left(\xi_{1}+\xi_{2}\right) z_{4}+N g_{1}, \\
\dot{z}_{2}= & -\frac{1}{2}\left(\xi_{1}-\xi_{2}\right) z_{1}-\frac{1}{2}\left(\xi_{1}+\xi_{2}\right) z_{2} \\
& +\frac{1}{2}\left(\xi_{1}+\xi_{2}\right) z_{3}-\frac{1}{2}\left(\xi_{1}+\xi_{2}\right) z_{4}+N g_{2}, \\
\dot{z}_{3}= & -2 z_{3}+\frac{1}{2}\left(\xi_{1}+\xi_{2}\right) z_{1}-\frac{1}{2}\left(\xi_{1}+\xi_{2}\right) z_{2} \\
& +\frac{1}{2}\left(\xi_{1}+\xi_{2}\right) z_{3}+\frac{1}{4}\left(\xi_{1}-\xi_{2}\right) z_{4}+N g_{3}, \\
\dot{z}_{4}= & -2 z_{4}+\frac{1}{2}\left(\xi_{1}+\xi_{2}\right) z_{1}+\frac{1}{2}\left(\xi_{1}+\xi_{2}\right) z_{2} \\
& -\frac{1}{2}\left(\xi_{1}-\xi_{2}\right) z_{3}+\frac{1}{2}\left(\xi_{1}+\xi_{2}\right) z_{4}+N g_{4},
\end{aligned}
$$

where the nonlinear functions $\mathrm{Ng}_{i}(i=1, \ldots, 4)$ are exhibited in the appendix.

Similar to Section 3.1.1, we can get the equations in the critical variables $z_{1}$ and $z_{2}$, up to leading orders, as follows:

$$
\begin{aligned}
\dot{z}_{1}= & {\left[-\frac{1}{2}\left(\xi_{1}+\xi_{2}\right)-\frac{1}{4}\left(\xi_{1}+\xi_{2}\right)^{2}\right] z_{1}+\frac{1}{2}\left(\xi_{1}-\xi_{2}\right) z_{2} } \\
& +\frac{3}{4} z_{1}^{3}-\frac{1}{2} z_{2}^{3}+\frac{1}{2} z_{1}^{2} z_{2}-\frac{1}{4} z_{2}^{2} z_{1},
\end{aligned}
$$



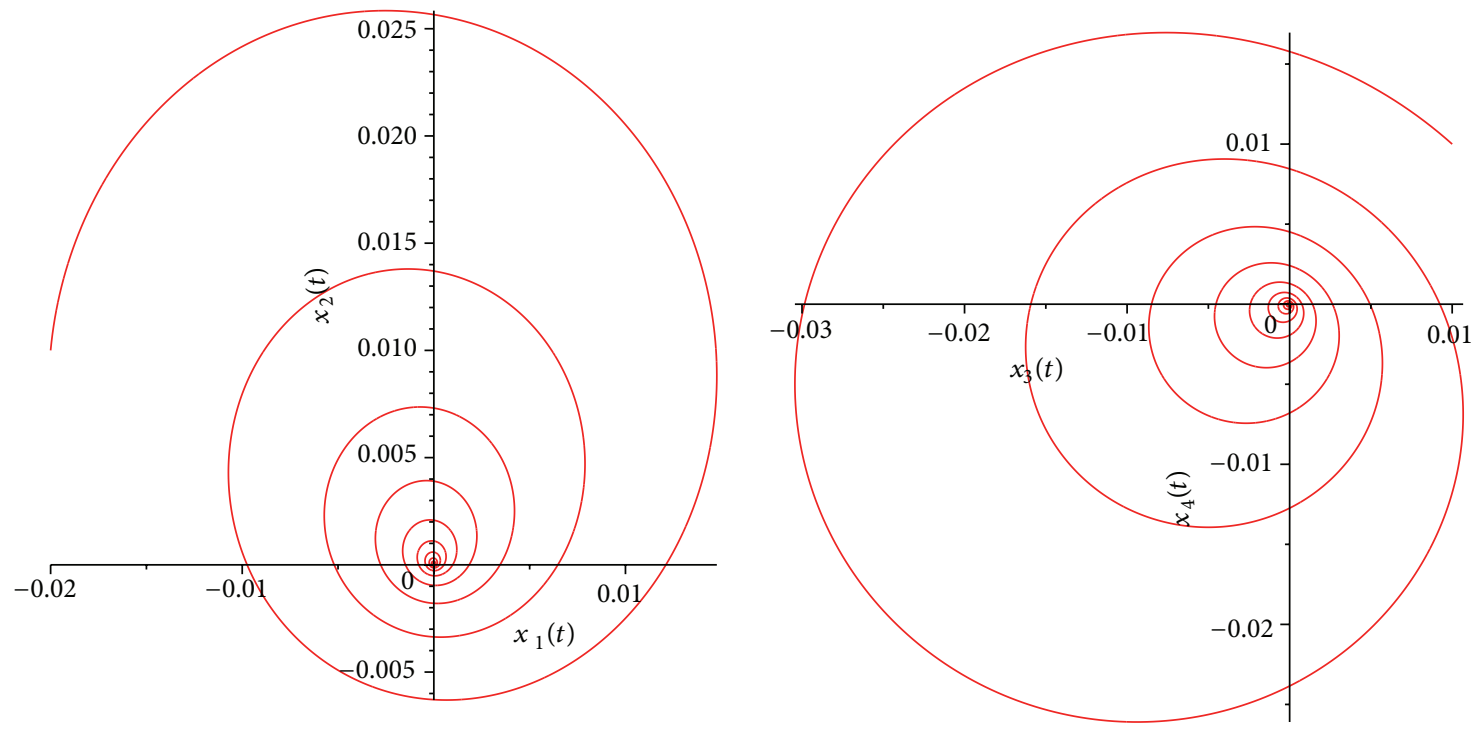

FIGURE 6: Trajectory projection starting from $\left(x_{1}, x_{2}, x_{3}, x_{4}\right)=(-0.02,0.01,0.01,0.01)$ converges to the E.S. when $\left(\delta_{1}, \delta_{2}\right)=(-0.2,-0.2)$.

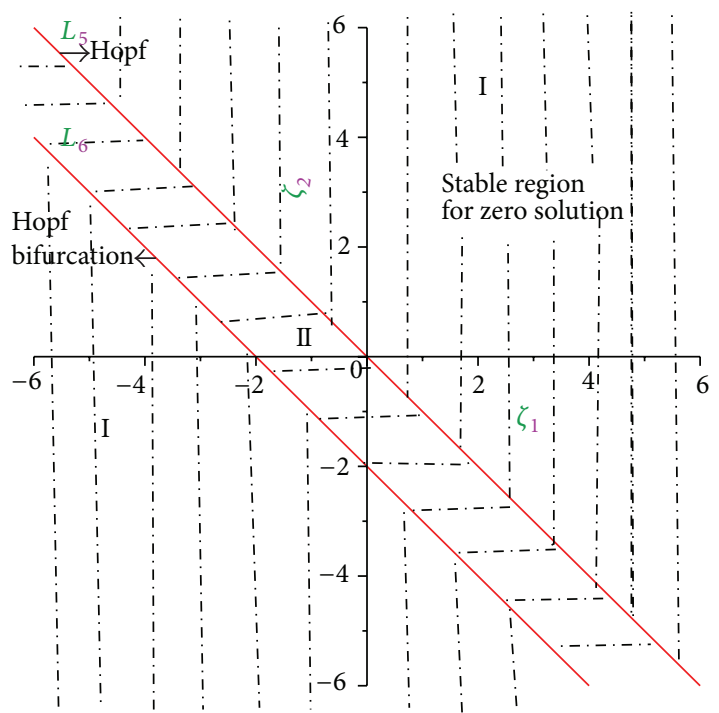

FIGURE 7: Bifurcation diagram on detuning parameters for the case of double zero and two negative eigenvalues.

$$
\begin{aligned}
\dot{z}_{2}= & -\frac{1}{2}\left(\xi_{1}-\xi_{2}\right) z_{1}+\left[-\frac{1}{2}\left(\xi_{1}+\xi_{2}\right)-\frac{1}{4}\left(\xi_{1}+\xi_{2}\right)^{2}\right] z_{2} \\
& +z_{1}^{3}-\frac{17}{4} z_{2}^{3}-\frac{5}{4} z_{1}^{2} z_{2}-4 z_{2}^{2} z_{1} .
\end{aligned}
$$

To find the stability conditions of the initial equilibrium solution $\left(z_{1}, z_{2}\right)=(0,0)$, one may evaluate the Jacobian matrix of $(24 \mathrm{a})-(24 \mathrm{~d})$ at $\left(z_{1}, z_{2}\right)=(0,0)$ and obtain

$$
\mathbf{J}_{\left(z_{i}=0\right)}=\left[\begin{array}{ll}
c_{11} & c_{12} \\
c_{21} & c_{21}
\end{array}\right]
$$

where

$$
\begin{aligned}
& c_{11}=-\frac{1}{2}\left(\xi_{1}+\xi_{2}\right)-\frac{1}{4}\left(\xi_{1}+\xi_{2}\right)^{2}, \\
& c_{12}=\frac{1}{2}\left(\xi_{1}-\xi_{2}\right), \\
& c_{21}=-\frac{1}{2}\left(\xi_{1}-\xi_{2}\right), \\
& c_{22}=-\frac{1}{2}\left(\xi_{1}+\xi_{2}\right)-\frac{1}{4}\left(\xi_{1}+\xi_{2}\right)^{2} .
\end{aligned}
$$

The characteristic polynomial is

$$
\begin{aligned}
f(\lambda)= & \lambda^{2}+\left[\left(\xi_{1}+\xi_{2}\right)+\frac{1}{2}\left(\xi_{1}+\xi_{2}\right)^{2}\right] \lambda \\
& +\left[\left[\frac{1}{2}\left(\xi_{1}+\xi_{2}\right)+\frac{1}{4}\left(\xi_{1}+\xi_{2}\right)^{2}\right]^{2}+\frac{1}{4}\left(\xi_{1}-\xi_{2}\right)^{2}\right] .
\end{aligned}
$$

The stability conditions for the initial equilibrium solution $\left(z_{1}, z_{2}\right)=(0,0)$ are

$$
\begin{gathered}
\left(\xi_{1}+\xi_{2}\right)+\frac{1}{2}\left(\xi_{1}+\xi_{2}\right)^{2}>0, \\
{\left[\frac{1}{2}\left(\xi_{1}+\xi_{2}\right)+\frac{1}{4}\left(\xi_{1}+\xi_{2}\right)^{2}\right]^{2}+\frac{1}{4}\left(\xi_{1}-\xi_{2}\right)^{2}>0 .}
\end{gathered}
$$

It is easy to see that $\left[(1 / 2)\left(\xi_{1}+\xi_{2}\right)+(1 / 4)\left(\xi_{1}+\xi_{2}\right)^{2}\right]^{2}+$ $(1 / 4)\left(\xi_{1}-\xi_{2}\right)^{2}>0$ unless $\left(\xi_{1}, \xi_{2}\right)=(0,0)$, so if $\left(\xi_{1}+\xi_{2}\right)+(1 / 2)\left(\xi_{1}+\xi_{2}\right)^{2}>0$, the initial equilibrium solution is stable. From this equality, one can denote

$$
\begin{gathered}
L_{5}: \xi_{1}+\xi_{2}=0, \\
L_{6}: \xi_{1}+\xi_{2}=-2 .
\end{gathered}
$$




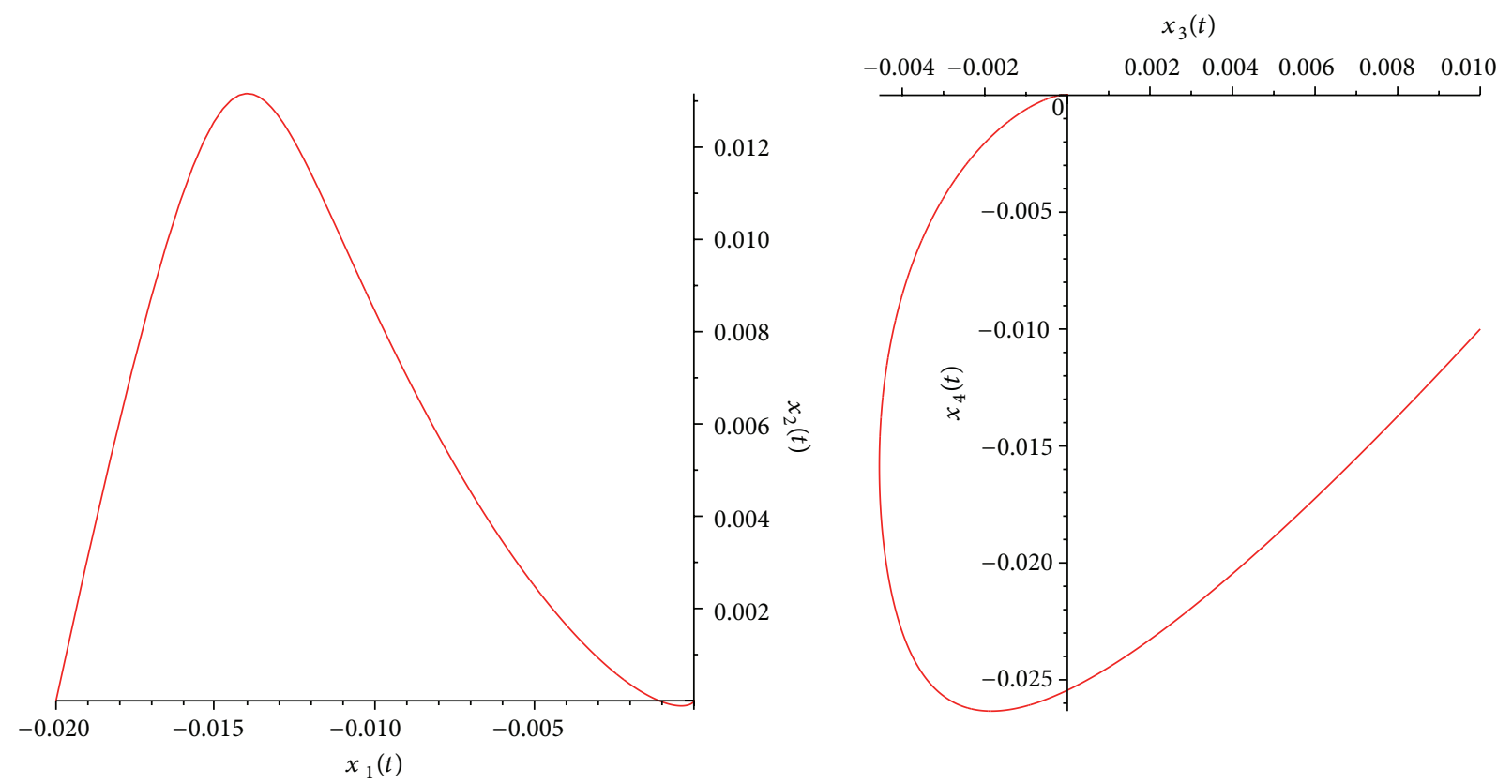

FIGURE 8: Trajectory projection starting from $\left(x_{1}, x_{2}, x_{3}, x_{4}\right)=(-0.02,0,0.01,-0.01)$ converges to the E.S. when $\left(\xi_{1}, \xi_{2}\right)=(0.1,0.2)$.
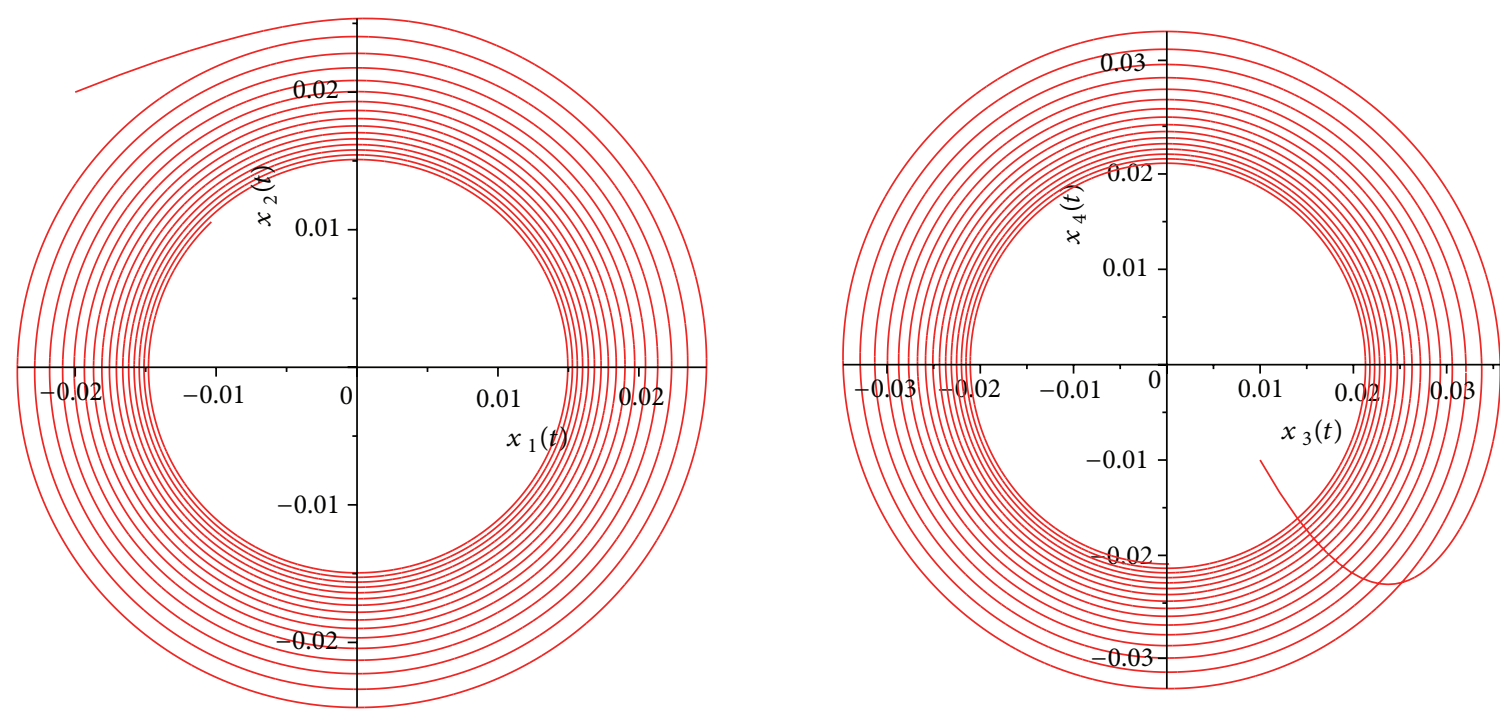

Figure 9: Trajectory projection starting from $\left(x_{1}, x_{2}, x_{3}, x_{4}\right)=(-0.02,0.02,0.01,-0.01)$ converges to the E.S. when $\left(\xi_{1}, \xi_{2}\right)=(0.2,-0.2)$.

For $\left(\xi_{1}, \xi_{2}\right) \in L_{5}$ or $\left(\xi_{1}, \xi_{2}\right) \in L_{6}$, Hopf bifurcation may occur. The discussion for Hopf bifurcation is similar to that of Section 3.1.1. The bifurcation diagram for this case is shown in Figure 7. It is observed from this figure that the critical curves $L_{5}, L_{6}$ separate $x_{1}-x_{2}$ plane into two parts. While $\left(\xi_{1}, \xi_{2}\right)$ is in area I, the initial equilibrium solution is stable. In area II, the initial equilibrium solution is unstable. Hopf bifurcation may occur along $L_{5}$ and $L_{6}$.

By choosing $\left(\xi_{1}, \xi_{2}\right)=(0.1,0.2)$, which represents a point in the parameter space located in the stable region I of the E.S., one can use the time integration to find a numerical solution. The trajectory starting from the initial point $\left(x_{1}, x_{2}, x_{3}, x_{4}\right)=(-0.02,0,0.01,-0.01)$ converges to the origin shown in Figure 8, implying that the E.S. is stable.

If one chooses $\left(\xi_{1}, \xi_{2}\right)=(0.2,-0.2)$, which is located in the line $L_{5}$, Hopf bifurcation may occur. The phase trajectories starting from $\left(x_{1}, x_{2}, x_{3}, x_{4}\right)=(-0.02,0.02,0.01,-0.01)$ are shown in Figure 9. Similar to the case of $\left(\xi_{1}, \xi_{2}\right) \in L_{5}$, the trajectories of $\left(\xi_{1}, \xi_{2}\right) \in L_{6}$ are omitted here.

3.2.2. Double Zero and a Pair of Pure Imaginary Eigenvalues. Corresponding to Section 3.1.2, take parameters $\mu_{1}=1, \mu_{2}=$ $-1, \sigma_{1}=1, \sigma_{2}=-1, \alpha=0, \beta=0, k_{1}=1, k_{2}=-2, a_{4}=a_{5}=$ $a_{6}=a_{7}=a_{8}=a_{9}=a_{10}=1, b_{6}=b_{7}=b_{8}=b_{9}=b_{10}=b_{11}=1$. 

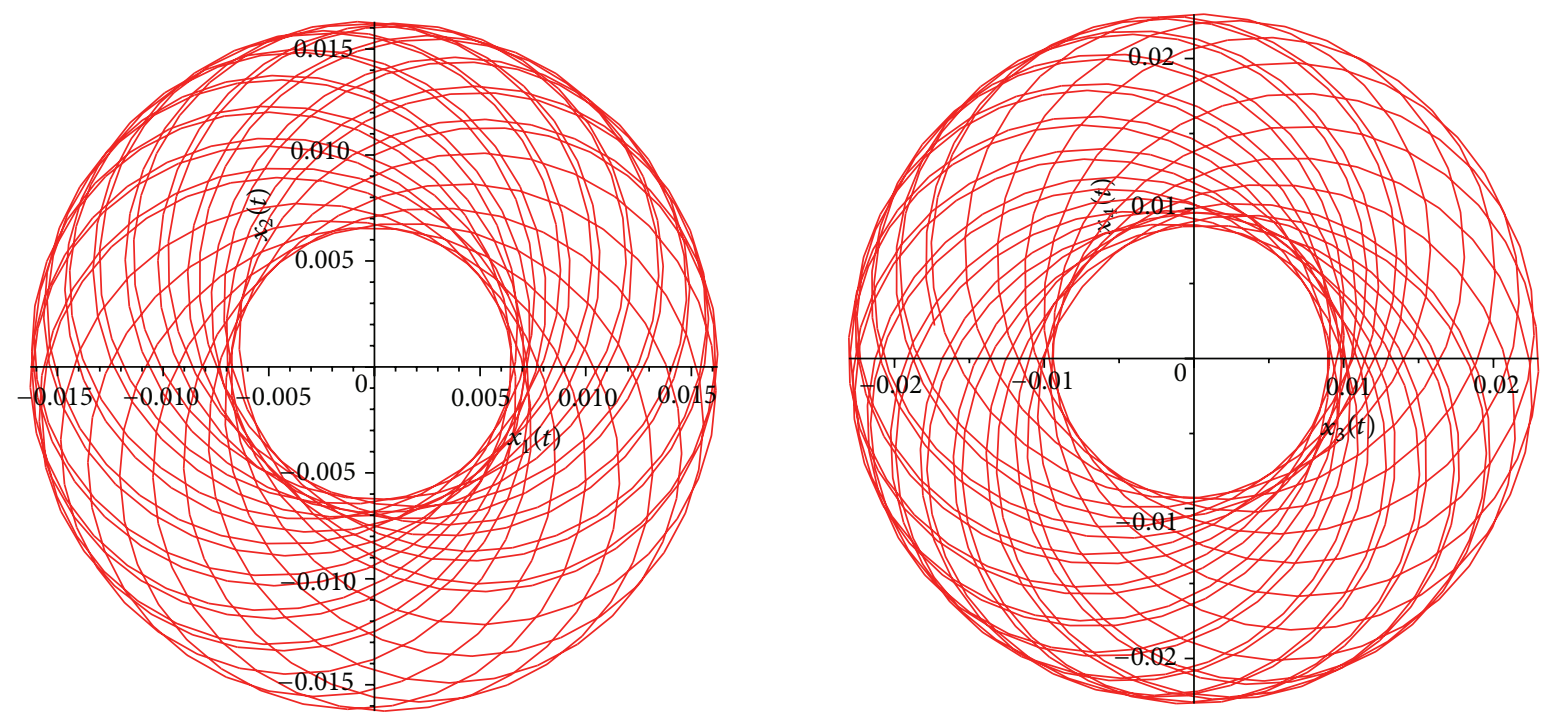

FIGURE 10: A two-dimensional torus starting from $\left(x_{1}, x_{2}, x_{3}, x_{4}\right)=(0,0.01,0,0.01)$ when $\left(\xi_{1}, \xi_{2}\right)=(-0.1,0.1)$.

It yields that $b_{2}=4, b_{1}=b_{3}=b_{4}=0$ and the Jacobian matrix (4) has the eigenvalues $\lambda_{1,2}=0, \lambda_{3,4}= \pm 2 i$.

Choosing $\sigma_{1}$ and $\sigma_{2}$ as perturbation parameters, and then using the parameter transformation $\sigma_{1}=1+\xi_{1}, \sigma_{2}=-1+\xi_{2}$, then characteristic polynomial (5) of the Jacobian matrix (4) becomes

$$
\widetilde{f}(\lambda)=\lambda^{4}+\widetilde{b}_{2} \lambda^{2}+\widetilde{b}_{3} \lambda+\widetilde{b}_{4}
$$

where

$$
\begin{aligned}
& \widetilde{b}_{2}=\xi_{1}^{2}+\xi_{2}^{2}+2 \xi_{1}-2 \xi_{2}+4, \\
& \widetilde{b}_{3}=2 \xi_{1}^{2}-2 \xi_{2}^{2}+4 \xi_{1}+4 \xi_{2}, \\
& \widetilde{b}_{4}=\xi_{2}^{2} \xi_{1}^{2}+2 \xi_{1}^{2}+2 \xi_{2}^{2}-2 \xi_{1}^{2} \xi_{2}+2 \xi_{2}^{2} \xi_{1} .
\end{aligned}
$$

Denote $L_{7}: 2 \xi_{1}^{2}-2 \xi_{2}^{2}+4 \xi_{1}+4 \xi_{2}=0$. It is easy to see that $\widetilde{b}_{4}>0$, unless $\left(\xi_{1}, \xi_{2}\right)=(0,0)$. One may get the following two facts:

(i) there exist no zero eigenvalues when $\left(\xi_{1}, \xi_{2}\right)=(0,0)$ since $\widetilde{b}_{4} \neq 0$;

(ii) when $\left(\xi_{1}, \xi_{2}\right) \in L_{7}$, except for $\xi_{1}=0$ or $\xi_{1}=-2$, there exist two pairs of pure imaginary eigenvalues; when $\left(\xi_{1}, \xi_{2}\right)=(0,0)$ or $\left(\xi_{1}, \xi_{2}\right)=(-2,2)$, there exist a double zero and a pair of pure imaginary eigenvalues.

In fact, supposing that there exist two pairs of pure imaginary eigenvalues, we have $\widetilde{b}_{3}=0$, which follows $\xi_{1}+\xi_{2}=$ 0 or $\xi_{1}-\xi_{2}+2=0$. For $\left(\xi_{1}, \xi_{2}\right) \in L_{7}$, the characteristic (31) becomes

$$
\lambda^{4}+\left(2 \xi_{1}^{2}+4\right) \lambda^{2}+\xi_{1}^{2}\left(\xi_{1}+2\right)^{2}=0
$$

The solutions of (33) are $\lambda_{1,2}= \pm \xi_{1} i, \lambda_{3,4}= \pm\left(\xi_{1}+2\right) i$. It is easy to show that if $\xi_{1}=0$ or $\xi_{1}=-2$, there exist a double zero and a pair of pure imaginary eigenvalues. For the other case, there always exist eigenvalues with positive real parts, so the initial equilibrium solution is unstable.

By the analytic study, we know that when $\left(\xi_{1}, \xi_{2}\right) \in L_{7}$, except for $\xi_{1}=0$ or $\xi_{1}=-2$, there exist two pairs of pure imaginary eigenvalues (including resonance and nonresonance cases). Choosing $\left(\xi_{1}, \xi_{2}\right)=(-0.1,0.1) \in L_{7}$, one can use time integration to find a numerical solution shown in Figure 10. A 2D torus is obtained when the trajectory starts from the initial point $\left(x_{1}, x_{2}, x_{3}, x_{4}\right)=(0,0.01,0,0.01)$, which implies that there is a $2 \mathrm{D}$ torus for two pairs of pure imaginary eigenvalues.

\section{Conclusions}

In this work, the stability of a simply supported functionally graded material rectangular plate subjected to the transversal and in-plane excitations has been studied in detail to show rich dynamical behaviors in the vicinity of critical points. Hao et al. [17] studied the bifurcation and chaos of FGM rectangular plate with numerical methods. We mainly investigate the local stability and bifurcation of the FGM plate in the vicinity of the equilibrium point using analytical methods. When the stable conditions for the initial equilibrium solution are not satisfied, bifurcations including Hopf bifurcation and $2 \mathrm{D}$ torus may occur. The stable conditions, stable regions, and critical bifurcation curves for the steady state solutions are presented explicitly in terms of the system parameters. With both analytical and numerical methods, bifurcation behaviors on damping parameters and detuning parameters are studied, respectively. Numerical computations have been performed and shown for each of the bifurcation cases. All numerical solutions agree with the analytical predictions. 


\section{Appendix}

The nonlinear functions $N f_{i}(i=1,2,3,4)$ in (3a)-(3d) are

$$
\begin{aligned}
N f_{1}= & a_{4} x_{1}^{2} x_{2}+a_{4} x_{2}^{3}+\left(a_{10}-a_{8}+a_{5}\right) x_{3}^{2} x_{2} \\
& +\left(-a_{10}+a_{8}+a_{5}\right) x_{4}^{2} x_{2}+2 a_{9} x_{1} x_{2} x_{3} \\
& +\left(a_{6}-a_{9}\right) x_{1}^{2} x_{4}+\left(a_{6}+a_{9}\right) x_{2}^{2} x_{4} \\
& +2\left(a_{8}+a_{10}\right) x_{1} x_{3} x_{4}+a_{7} x_{3}^{2} x_{4}+a_{7} x_{4}^{3}, \\
N f_{2}= & -a_{4} x_{1}^{2} x_{2}-a_{4} x_{1}^{3}-\left(a_{10}+a_{8}+a_{5}\right) x_{3}^{2} x_{1} \\
& -\left(-a_{10}-a_{8}+a_{5}\right) x_{4}^{2} x_{1}-2 a_{9} x_{1} x_{2} x_{4} \\
& -\left(a_{6}+a_{9}\right) x_{1}^{2} x_{3}-\left(a_{6}-a_{9}\right) x_{2}^{2} x_{3} \\
& -2\left(a_{8}-a_{10}\right) x_{2} x_{3} x_{4}-a_{7} x_{4}^{2} x_{3}-a_{7} x_{3}^{3}, \\
N f_{3}= & b_{8} x_{1}^{2} x_{2}+\left(b_{6}-b_{10}\right) x_{1}^{2} x_{4}+\left(b_{6}+b_{10}\right) x_{2}^{2} x_{4} \\
& +b_{8} x_{2}^{3}-\left(b_{11}-b_{9}\right) x_{3}^{2} x_{2}+\left(b_{11}+b_{9}\right) x_{4}^{2} x_{2} \\
& +2 b_{10} x_{1} x_{2} x_{3}+2 b_{11} x_{1} x_{3} x_{4}+b_{7} x_{3}^{2} x_{4}+b_{7} x_{4}^{3}, \\
N f_{4}= & -b_{8} x_{2}^{2} x_{1}-\left(b_{6}+b_{10}\right) x_{1}^{2} x_{3}+\left(b_{6}-b_{10}\right) x_{2}^{2} x_{3} \\
& -b_{8} x_{1}^{3}-\left(b_{11}+b_{9}\right) x_{3}^{2} x_{1}+\left(-b_{11}+b_{9}\right) x_{4}^{2} x_{1} \\
& -2 b_{10} x_{1} x_{2} x_{4}-2 b_{11} x_{2} x_{3} x_{4}-b_{7} x_{4}^{2} x_{3}-b_{7} x_{3}^{3} .
\end{aligned}
$$

The nonlinear functions $N g_{i}(i=1,2,3,4)$ in $(9 \mathrm{a})-(9 \mathrm{~d})$ are

$$
\begin{aligned}
N g_{1}= & \frac{9}{2} z_{1} z_{2} z_{4}+2 z_{1} z_{3} z_{4}+\frac{1}{2} z_{1} z_{2} z_{3}+\frac{1}{2} z_{1}^{2} z_{2}+\frac{5}{4} z_{1}^{2} z_{4} \\
& -\frac{1}{4} z_{2}^{2} z_{1}+\frac{3}{2} z_{2}^{2} z_{3}-\frac{3}{2} z_{3}^{2} z_{2}-\frac{5}{4} z_{3}^{2} z_{4}+\frac{1}{4} z_{4}^{2} z_{1} \\
& +\frac{7}{2} z_{4}^{2} z_{3}-\frac{13}{4} z_{2}^{2} z_{4}-\frac{9}{4} z_{4}^{2} z_{2}+\frac{5}{2} z_{1}^{2} z_{3} \\
& +\frac{7}{4} z_{3}^{2} z_{1}-\frac{1}{2} z_{2}^{3}-\frac{9}{4} z_{4}^{3}+\frac{3}{4} z_{1}^{3}+\frac{1}{2} z_{3}^{3}, \\
\mathrm{Ng}_{2}= & -\frac{13}{2} z_{1} z_{2} z_{4}+\frac{5}{2} z_{1} z_{3} z_{4}+3 z_{1} z_{2} z_{3}+9 z_{2} z_{3} z_{4} \\
& -\frac{5}{4} z_{1}^{2} z_{2}-z_{1}^{2} z_{4}-3 z_{4}^{2} z_{1}-13 z_{2}^{2} z_{4} \\
& +\frac{15}{4} z_{2}^{2} z_{3}-\frac{3}{4} z_{3}^{2} z_{2}-28 z_{3}^{2} z_{4}-\frac{53}{4} z_{4}^{2} z_{2}-4 z_{2}^{2} z_{1} \\
& +\frac{25}{4} z_{4}^{2} z_{3}+\frac{9}{4} z_{1}^{2} z_{3}-z_{3}^{2} z_{1}-\frac{17}{4} z_{2}^{3}-5 z_{4}^{3}+z_{1}^{3}-\frac{3}{4} z_{3}^{3}, \\
& -\frac{9}{2} z_{1} z_{2} z_{4}+\frac{1}{2} z_{1} z_{3} z_{4}+2 z_{1} z_{2} z_{3}-\frac{5}{4} z_{1}^{2} z_{2} \\
& -\frac{3}{2} z_{1}^{2} z_{4}-\frac{3}{2} z_{4}^{2} z_{1}-\frac{9}{2} z_{2}^{2} z_{4}-\frac{1}{4} z_{2}^{2} z_{3}+\frac{5}{4} z_{3}^{2} z_{2} \\
N g_{3}= &
\end{aligned}
$$

$$
\begin{aligned}
& +\frac{1}{2} z_{3}^{2} z_{4}-\frac{13}{4} z_{4}^{2} z_{2}-\frac{7}{2} z_{2}^{2} z_{1}+\frac{1}{4} z_{4}^{2} z_{3}-\frac{7}{4} z_{1}^{2} z_{3} \\
& -\frac{5}{2} z_{3}^{2} z_{1}-\frac{9}{4} z_{2}^{3}-\frac{1}{2} z_{4}^{3}-\frac{1}{2} z_{1}^{3}-\frac{3}{4} z_{3}^{3}, \\
N g_{4}= & 9 z_{1} z_{2} z_{4}-3 z_{1} z_{3} z_{4}-\frac{5}{2} z_{1} z_{2} z_{3}-\frac{13}{2} z_{2} z_{3} z_{4}+2 z_{1}^{2} z_{2} \\
& +\frac{3}{4} z_{1}^{2} z_{4}+\frac{15}{4} z_{4}^{2} z_{1}+\frac{53}{4} z_{2}^{2} z_{4}-3 z_{2}^{2} z_{3} \\
& +z_{3}^{2} z_{2}+\frac{5}{4} z_{3}^{2} z_{4}+13 z_{4}^{2} z_{2}+\frac{25}{4} z_{2}^{2} z_{1}-4 z_{4}^{2} z_{3}-z_{1}^{2} z_{3} \\
& +\frac{9}{4} z_{3}^{2} z_{1}+5 z_{2}^{3}+\frac{17}{4} z_{4}^{3}-\frac{13}{4} z_{1}^{3}+z_{3}^{3} .
\end{aligned}
$$

\section{Conflict of Interests}

The authors declare that there is no conflict of interests regarding the publication of this paper.

\section{Acknowledgments}

This work is supported by the National Natural Science Foundation of China (11172125) and the National Research Foundation for the Doctoral Program of Higher Education of China (20133218110025).

\section{References}

[1] M. Koizumi, "The concept of FGM," in Ceramic Transactions, vol. 34 of Functionally Gradient Materials, pp. 3-10, 1993.

[2] M. Yamanoushi, M. Koizumi, T. Hiraii, and I. Shiota, Proceedings of the First International Symposium on Functionally Gradient Materials, Sendai, Japan, 1990.

[3] G. N. Praveen and J. N. Reddy, "Nonlinear transient thermoelastic analysis of functionally graded ceramic-metal plates," International Journal of Solids and Structures, vol. 35, no. 33, pp. 4457-4476, 1998.

[4] P. J. Holmes, "Bifurcations to divergence and flutter in flowinduced oscillations: a finite dimensional analysis," Journal of Sound and Vibration, vol. 53, no. 4, pp. 471-503, 1977.

[5] X. L. Yang and P. R. Sethna, "Local and global bifurcations in parametrically excited vibrations of nearly square plates," International Journal of Non-Linear Mechanics, vol. 26, no. 2, pp. 199-220, 1991.

[6] P. Yu, W. Zhang, and Q. Bi, "Vibration analysis on a thin plate with the aid of computation of normal forms," International Journal of Non-Linear Mechanics, vol. 36, no. 4, pp. 597-627, 2001.

[7] M. Ye, Y. Sun, W. Zhang, X. Zhan, and Q. Ding, "Nonlinear oscillations and chaotic dynamics of an antisymmetric cross-ply laminated composite rectangular thin plate under parametric excitation," Journal of Sound and Vibration, vol. 287, no. 4-5, pp. 723-758, 2005.

[8] W. Zhang, Z. Yao, and M. Yao, "Periodic and chaotic dynamics of composite laminated piezoelectric rectangular plate with one-to-two internal resonance," Science in China E: Technological Sciences, vol. 52, no. 3, pp. 731-742, 2009. 
[9] S. I. Chang, A. K. Bajaj, and C. M. Krousgrill, "Non-Linear vibrations and chaos in harmonically excited rectangular plates with one-to-one internal resonance," Nonlinear Dynamics, vol. 4, no. 5, pp. 433-460, 1993.

[10] W. Zhang, C. Song, and M. Ye, "Further studies on nonlinear oscillations and chaos of a symmetric cross-ply laminated thin plate under parametric excitation," International Journal of Bifurcation and Chaos in Applied Sciences and Engineering, vol. 16, no. 2, pp. 325-347, 2006.

[11] A. Maccari, "The asymptotic perturbation method for nonlinear continuous systems," Nonlinear Dynamics, vol. 19, no. 1, pp. 1-18, 1999.

[12] A. Maccari, "Multiple resonant or non-resonant parametric excitations for nonlinear oscillators," Journal of Sound and Vibration, vol. 242, no. 5, pp. 855-866, 2001.

[13] W. Zhang, Y. Hao, X. Guo, and L. Chen, "Complicated nonlinear responses of a simply supported FGM rectangular plate under combined parametric and external excitations," Meccanica, vol. 47, no. 4, pp. 985-1014, 2012.

[14] P. Yu and K. Huseyin, "A perturbation analysis of interactive static and dynamic bifurcations," Institute of Electrical and Electronics Engineers. Transactions on Automatic Control, vol. 33, no. 1, pp. 28-41, 1988.

[15] K. Huseyin and P. Yu, "On bifurcations into nonresonant quasiperiodic motions," Applied Mathematical Modelling, vol. 12, no. 2, pp. 189-201, 1988.

[16] P. Yu, "Analysis on double Hopf bifurcation using computer algebra with the aid of multiple scales," Nonlinear Dynamics, vol. 27, no. 1, pp. 19-53, 2002.

[17] Y. X. Hao, L. H. Chen, W. Zhang, and J. G. Lei, "Nonlinear oscillations, bifurcations and chaos of functionally graded materials plate," Journal of Sound and Vibration, vol. 312, no. 4-5, pp. 862-892, 2008.

[18] X. Zhang, F. Chen, and H. Zhang, "Stability and local bifurcation analysis of functionally graded material plate under transversal and in-plane excitations," Applied Mathematical Modelling: Simulation and Computation for Engineering and Environmental Systems, vol. 37, no. 10-11, pp. 6639-6651, 2013. 


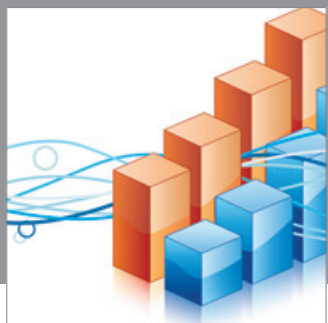

Advances in

Operations Research

mansans

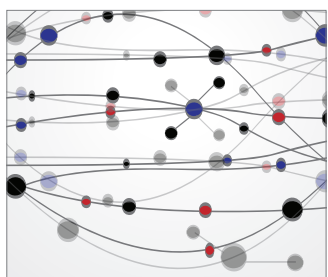

The Scientific World Journal
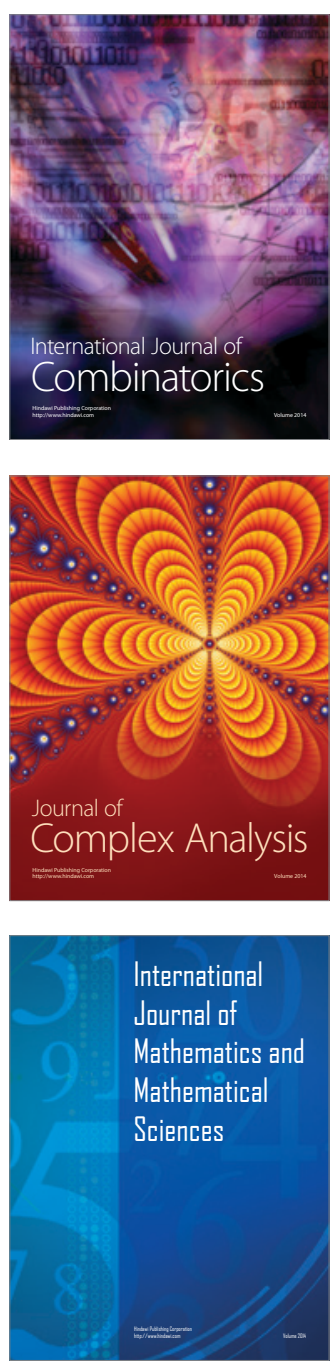
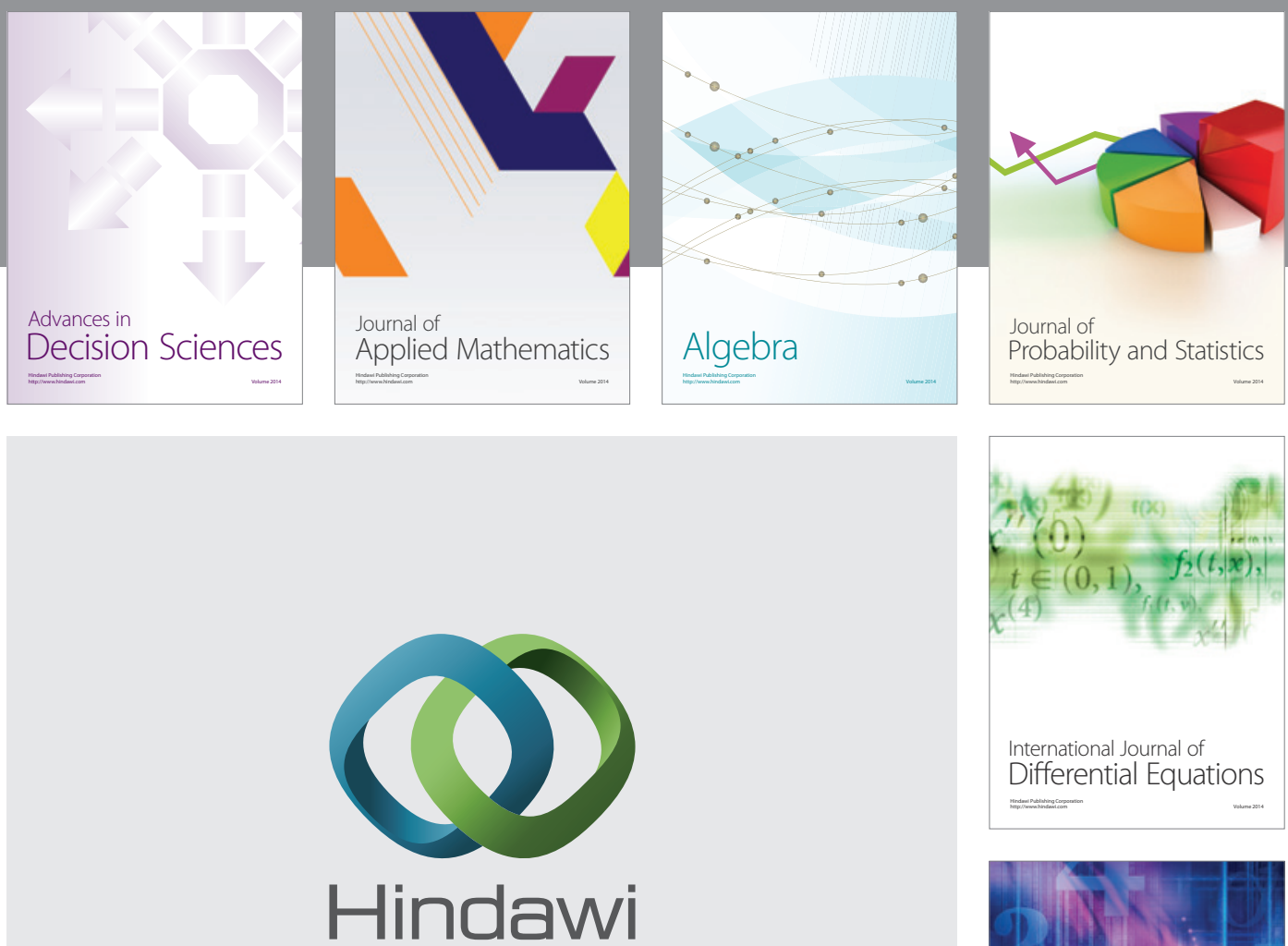

Submit your manuscripts at http://www.hindawi.com
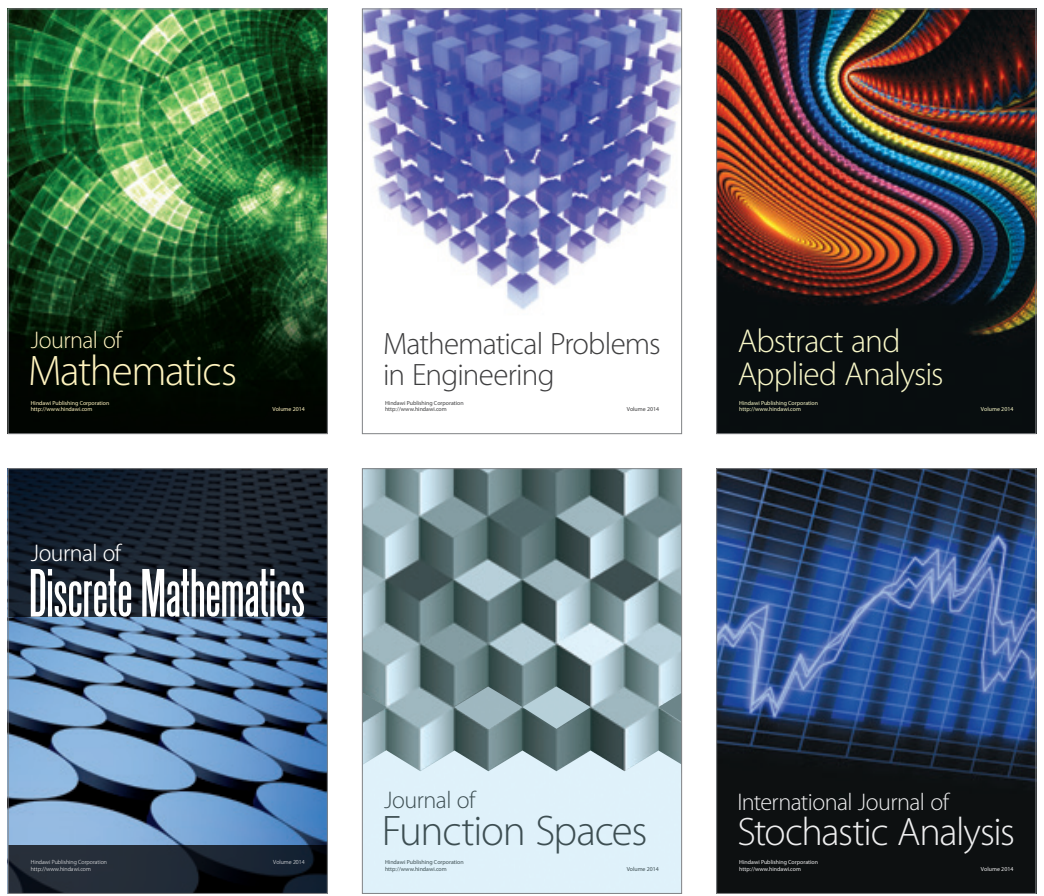

Journal of

Function Spaces

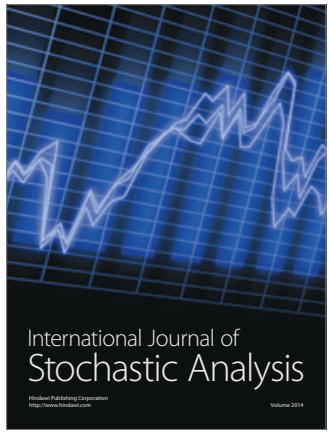

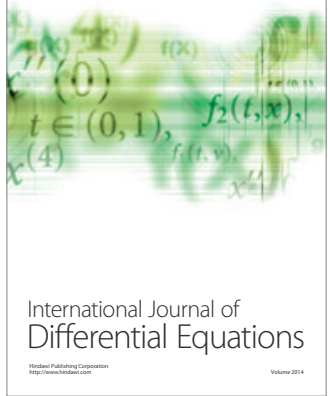
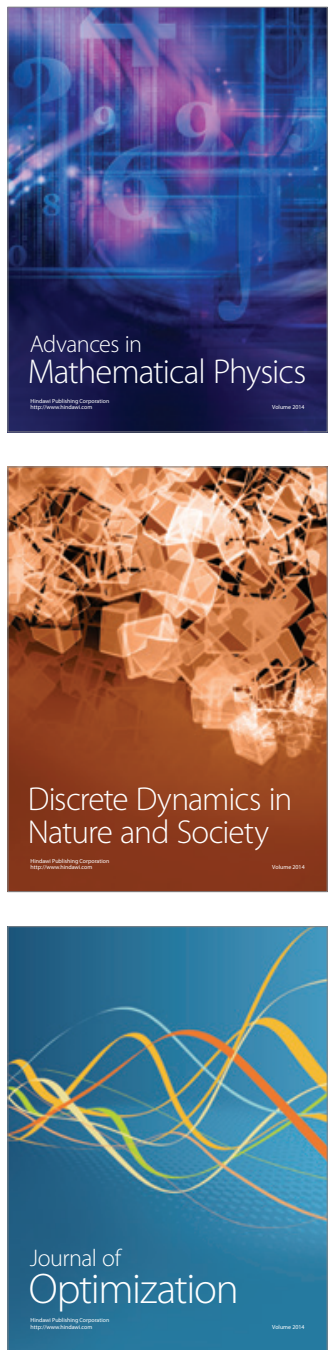